\title{
Ovarian hormone-induced beta-cell hypertrophy contributes to the homeostatic control of beta-cell mass in OLETF female rat, a model of Type II diabetes
}

\author{
M.Zhu ${ }^{1}$, A.Mizuno ${ }^{1}$, M.Kuwajima ${ }^{1}$, T.Ogino ${ }^{1}$, T.Murakami ${ }^{1}$, Y.Noma ${ }^{1}$, T.Sano ${ }^{2}$, K.Shima ${ }^{1}$ \\ ${ }^{1}$ Department of Laboratory Medicine, School of Medicine, University of Tokushima, Tokushima-City, Japan \\ ${ }^{2}$ Department of Pathology, School of Medicine, University of Tokushima, Tokushima-City, Japan
}

\begin{abstract}
Summary A sexual dimorphism regarding the incidence of diabetes mellitus in OLETF rat, a model of Type II diabetes, has been reported. As a result, the effects of ovarian hormones on beta cells per se was examined by comparing the capacity of beta-cell proliferation and changes in blood glucose and plasma insulin concentrations after a $70 \%$ pancreatectomy. All female animals were randomly assigned to two protocols. The rats involved in protocol I received either a $70 \%$ pancreatectomy (Px) or a sham pancreatectomy (sham) at 6 weeks of age, along with their diabetes-resistant counterparts, female LETO rats, which served as normal controls. The rats belonging to protocol II were given an ovariectomy (Ox) at 5 weeks of age, and one week later, they were subjected to either Px or the sham operation, with/without hormone (estradiol, $50 \mu \mathrm{g} / \mathrm{kg}$; testosterone, $1 \mathrm{mg} / \mathrm{kg}$ ) replacement. The findings indicate that the capacity for compensatory growth of beta cells after Px was affected by both sex hormonal and genetic components, since a $70 \%$ Px resulted in sustained hyperglycaemia within the first week after surgery, but was ameliorated by
\end{abstract}

an increase in beta-cell mass thereafter in the non-Ox Px OLETF rats. The Ox also caused a decline in beta-cell mass which could be improved by replacement with ovarian hormones. Not only endogenous but also replacement ovarian hormones, led to a beneficial effect on beta cells per se in OLETF female rats. This was reflected by an increased beta-cell mass accompanied by a parallel increase in plasma immunoreactive insulin concentration. The effects of ovarian hormones, however, contributed to the beta-cell hypertrophy rather than expansion of the beta-cell population to achieve glucose homeostasis, as evidenced by an increased area of individual beta-cell after Px rather than an increased BrdU-labelling index for the beta cells. The present study suggests that ovarian hormone-induced beta-cell hypertrophy may typically occur, to compensate for changes in functional demand as the results of a $70 \%$ Px in female OLETF rats. [Diabetologia (1998) 41: 799-805]

Keywords Ovarian hormones, beta-cell, hypertrophy, pancreatectomy, OLETF rat.
Type II (non-insulin-dependent) diabetes mellitus model rats, namely the Otsuka Long-Evans Tokushima Fatty (OLETF) show sexual dimorphism with re-

Received: 17 November 1997 and in revised form 9 February 1998

Corresponding author: Dr. K. Shima, MD, Ph.D, Department of Laboratory Medicine, School of Medicine, University of Tokushima, 3-18-15, Kuramoto-Cho, Tokushima, 770-8503, Japan Abbreviations: OLETF, Otsuka Long-Evan Tokushima Fatty; LETO, Long-Evans-Tokushima-Otsuka; Px, pancreatectomy; Sham, sham-pancreatectomy; BrdU, bromodeoxyuridine; LI, labelling index; Ox, ovariectomy; IRI, immunoreactive insulin. spect to the incidence of diabetes mellitus, with the cumulative incidences of diabetes in male and female OLETF rats being $86 \%$ and $0 \%$, at ages over 23 weeks [1]. However, the females are also potentially diabetic because approximately $40 \%$ of offspring hybrid $\mathrm{F} 1$ male rats (OLETF ox LETO ô, Long-Evans-Tokushima-Otsuka, a counterpart strain of diabetes-resistance rats) also develop diabetes [2]. In this case, an early ovariectomy (Ox) of some led to the development of diabetes [3], suggesting a hormonal role in this sexual dimorphism. Ovarian hormones have been shown to affect beta-cell function and glucose homeostasis by increasing insulin pro- 
duction [4] and acting synergistically with insulin to increase glucose uptake into cells [5, 6]. An important remaining issue is the effects of ovarian hormones on beta cells per se, particularly, with respect to alteration in beta-cell mass, since this alteration is a major determinant of the quantity of insulin which is secreted and ordinarily varies, depending on the degree of diabetogenic factors, such as obesity and insulin resistance which are present [7]. Only a few studies have examined these effects. In addition, the extent to which ovarian hormones protect diabetes through such alterations is largely not known. In a recent study, we reported the failure of pancreatic beta-cell proliferation in male OLETF rats, induced by a $70 \%$ pancreatectomy [8], as a cause of sustained hyperglycaemia. This may represent a critical pathogenetic event prior to the onset of overt diabetes. In conjuction with this, it would be interesting to determine if the loss of endogenous ovarian hormones by Ox contributes to this defect in beta-cell proliferation in the female OLETF rats, and, if so, whether this defect can be reversed by ovarian hormone replacement. To clarify the effects of ovarian hormones on beta cells per se, we report a study using partially pancreatectomized (Px) female OLETF rats with or without an early Ox, along with their diabetes-resistance counterpart (LETO) female rats as normal controls. The study is an examination of the effects of ovarian hormones on beta cells per se by comparing changes in blood glucose, plasma insulin concentration, beta-cell labelling index (LI) and beta-cell mass.

\section{Materials and methods}

Animals. Female OLETF and their diabetes-resistance counterpart LETO rats, aged 4 weeks, were obtained from the Tokushima Research Institute, Otsuka Pharmaceutical Co. and were maintained in our animal facilities under specific pathogen-free conditions (Institute of Animal Experimentation, Tokushima University). The temperature $\left(21 \pm 2{ }^{\circ} \mathrm{C}\right)$, humidity $(55 \pm 5 \%)$ and lighting (07:00-19:00 h) were all strictly controlled. The animals were supplied with water ad libitum and standard rat chow (Oriental Yeast, Tokyo, Japan).

Experimental design. Experiments were carried out using two protocols. Protocol I was performed on rats at 6 weeks of age, in which all female rats were allocated at random to two groups, partial pancreatectomy (Px) and sham-pancreatectomy (sham), along with LETO female rats as normal controls. Each group was divided into 2 subgroups, based on the date of killing after surgery, a 7-day group and a 28-day group. For Protocol II, all female OLETF rats were castrated by Ox under sodium pentobarbitone anesthesia at 5 weeks of age. A week later (6 weeks of age), they randomly received either a partial Px or a sham. The Px rats were divided into three subgroups (I, II and III), based on the treatment received after surgery. The animals from groups I and II were treated with subcutaneous replacement doses of either estradiol valerate at $50 \mu \mathrm{g} / \mathrm{kg}$ BW at $48 \mathrm{~h}$ intervals (Shering Japan, Tokyo, Japan) or testosterone enanthate at $1 \mathrm{mg} / \mathrm{kg} \mathrm{BW}$, at weekly intervals (Sher- ing Japan, Tokyo, Japan). Bioactivity of the hormone product used here and the relative insensitivity of the strain of the rats to the product dictated the dosage and the protocol of administration. The animals from group III and the sham subjects received an equal volume of vehicle only (no hormone). Injections were initiated on day 3 after surgery and continued until 1 week prior to killing.

$70 \%$ pancreatectomy. After an overnight starvation, the animals were anesthetized with ether and given additional ether, if required, during surgery. All pancreatic tissue was removed by gentle abrasion with cotton applicators, except for the anatomically well-defined remnant bordered by the branch of the hepatic portal vein and the first portion of the duodenal loop. The sham operation was performed by disengaging the pancreas from the mesentery and gently rubbing it between the fingers. All operations were performed by one person, whose surgical technique was well controlled, and as a result, the samples of the removed segments from each group gave an expected coefficient variation of $10 \%$ or less. At that time (6 weeks of age), the remnant equivalent of the pancreas constituted $29.6 \pm 2.3 \%(n=6)$ of the total pancreatic weight in the female OLETF rats and $30.1 \pm 3.1 \%(n=4)$ in the female LETO rats. During the first week after surgery, body weight and non-fasting blood glucose concentrations were measured daily at 16.00-17.00 hours and thereafter once per week at the same time of day.

Tissue processing. At each experimental time point, the animals were starved overnight, and killed under deep anesthesia, except for the 7-day group, which were intraperitoneally injected with bromodeoxyuridine (BrdU, Boehringer Mannheim $\mathrm{GmbH}$, Mannheim, Germany) dissolved in phosphate-buffered saline, at a dose of $100 \mathrm{mg} / \mathrm{kg} \mathrm{BW} 4 \mathrm{~h}$ before being killed [9]. The abdomen was quickly opened, and blood was withdrawn from the aorta for determination of immunoreactive insulin (IRI). Each pancreas or Px remnant was excised and cleared of extraneous lymph nodes and fat. The pancreata from the sham rats were divided into two portions, the remnant equivalent and the remainder of the pancreas. The remnant equivalent is that portion of the pancreas with the same anatomical boundaries as the remnant left in the $70 \%$ Px rats. Individual tissue samples were then weighed and cassetted in identical anatomic orientations, and then placed in Bouin's fixative. After fixation, the tissues were embedded in paraffin according to standard protocols.

Assay. Blood samples were obtained by tail snipping, and nonfasting blood glucose values were determined using the glucose oxidase method (Toecho Super, Kyoto Daiichi Kagaku Co., Ltd., Kyoto, Japan). Plasma IRI measurement was determined by a commercially available IRI kit (Eiken Kagaku Co., Tokyo, Japan) with rat insulin (Novo, Bagsvared, Denmark) as a standard.

Quantitative morphometrics. Four sets of three serial sections (3-5 $\mu \mathrm{m}$ thick) were obtained at intervals of about $250 \mu \mathrm{m}$. The sections were first deparaffined, and then immunostained using commercial ABC kits (Amersham, UK) for insulin and BrdU-insulin double immunostaining studies. The primary antibodies used were polyclonal anti-porcine insulin antibody (1:400, Dako Carpinteria, CA, USA) and monoclonal antiBrdU antibody obtained from a cell proliferation kit (Amersham, Arlington Heights, Ill., USA). The sections intended for the double-labelling study were first immunostained for BrdU and developed with 3,3'-diaminobenzidine. The same sections were then stained for insulin and visualized by an al- 

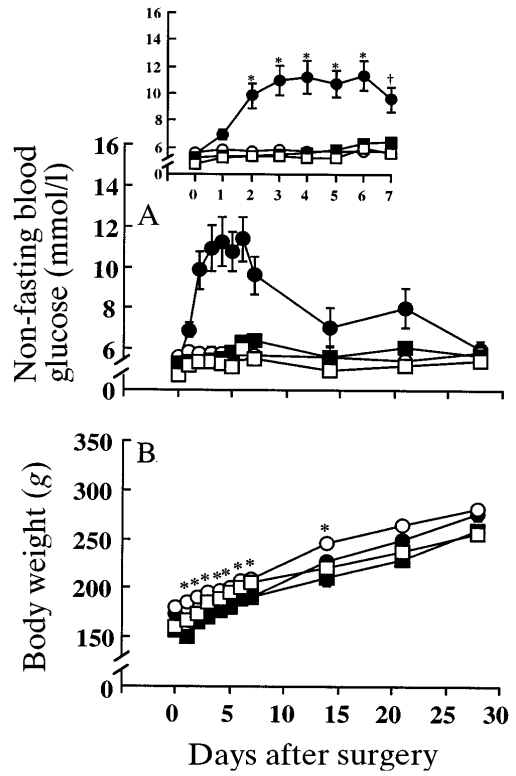

Fig. 1. Effects of a $70 \%$ Px on non-fasting blood glucose (A) and body weight (B) in Px OLETF (-), sham OLET $(\bigcirc-\bigcirc)$, Px LETO ( $-\square)$ and sham LETO $(\square-\square)$ rats during the period of observation. Points and bars are expressed as means \pm SEM. Values shown are for all surviving rats in each group, the number of which decreased with time as rats were killed from each subgroup at 7 and 28 days after surgery. Inset represents data for the non-fasting blood glucose using a magnified scale for the first 7 days after surgery of the $\mathrm{x}$-axis. $\dagger p<0.05,{ }^{*} p<0.01$ vs all other groups

kaline phosphatase substrate (Vector Red, Vector Laboratories Inc., Burlingame, CA, USA) as the chromogen. Using Weibel's point-counting morphometrics [10], the relative volumes (\%) of beta cells and total islets cells were quantitated at the original $200 \times$ magnification on a monitor screen using an Olympus microscope connected to a colour video camera and an image analysis system. Starting at a random point, in one corner of the section, the relative volumes (\%) of beta cells and total islet cells were scored in every other field using a 96point grid with a minimum of 9600 points in 100 fields counted per tissue block. The relative volumes of both beta cells and total islet cells were calculated as the number of intercepts over that relevant tissue as a proportion of the total counts over pancreatic tissue. The intercepts over blood vessels, fat, ducts or interlobular spaces were subtracted to give the total pancreatic counts. To obtain absolute mass $(\mathrm{mg})$, the relative volume (\%) for each pancreatic portion was multiplied by the weight of that portion. Values for the two portions from sham rat (the remnant equivalent and the remainder of the pancreas) were summed to obtain the value for each rat. For beta-cell proliferation, BrdU-labelled nuclei were counted at an original magnification of $400 \times$. For each block, a minimum of $500 \mathrm{nu}-$ clei in the insulin-immunoreactive area were counted. The number of cells which were positive for both insulin and BrdU was determined and expressed as the percentage of BrdU labelling index for beta cells in the Px remnant or remnant equivalent pancreas, with extrapolation to the rate per $24 \mathrm{~h}$. The mean cross-sectional area of beta cells stained by anti-insulin antibody was measured in 20 randomly selected islets per block, and beta-cell nuclei were counted within this area at an original magnification of $400 \times$. The mean cross-sectional area of beta cells was then divided by the number of nu-
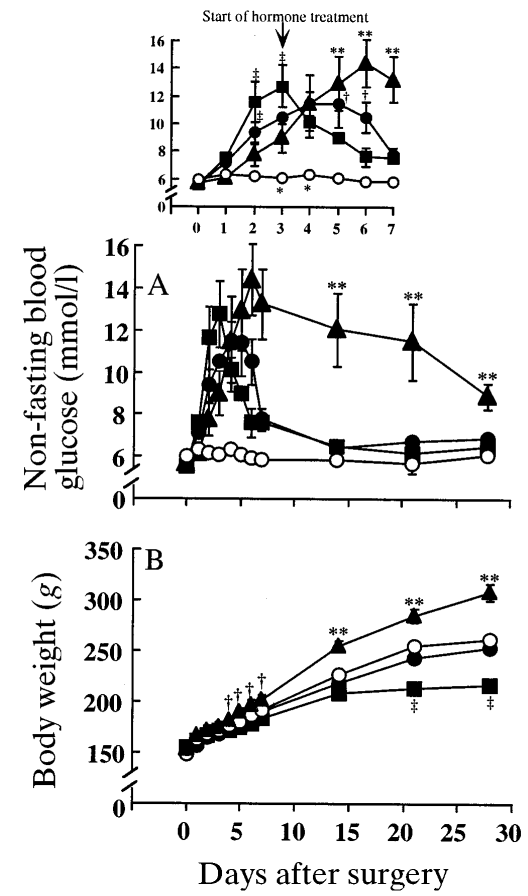

Fig. 2. Effects of a $70 \% \mathrm{Px}$ and an early Ox with or without hormone replacement on non-fasting blood glucose (A) and body weight (B) in the Ox-Px (-), Ox-Px + estradiol $(\square-\square)$ Ox-Px + testosterone $(\boldsymbol{\Delta}-\mathbf{\Delta})$ and Ox-sham $(\bigcirc-\bigcirc)$ OLETF rats after Px. All data are expressed as means \pm SEM. The protocol used and doses of estradiol and testosterone, as well as the animals used for data are the same as for Table 1 . Inset represents data for the non-fasting blood glucose using a magnified scale for the first 7 days after surgery of the $\mathrm{x}$-axis. $* p<0.05, * * p<0.01$ vs all other rats; $\dagger p<0.05$ vs both Ox-Px + estradiol and Ox-sham (A) and vs Ox-Px + estradiol group (B); $\neq p<0.05$ vs both $\mathrm{Ox}-\mathrm{Px}+$ testosterone and $\mathrm{Ox}-$ sham rats $(\mathrm{A})$ and $p<0.01$ vs both $\mathrm{Ox}-\mathrm{Px}$ and $\mathrm{Ox}$-sham rats (B)

clei to obtain the individual beta-cell area. The images were calibrated using images of a stage micrometer taken at the appropriate magnifications. All observations were made by one person.

Presentation of data and statistics. All results are expressed as means \pm SEM. The significance of differences was determined using one-way analysis of variance (ANOVA). Statistical calculations were performed using a commercial software package, StatView (Abacus Concepts/Brain Power Berkeley, Calif., USA). Differences were deemed to be significant when $p$ was less than 0.05 .

\section{Results}

Non-fasting blood glucose and body weight. Figure 1 shows changes in non-fasting blood glucose concentrations (Fig. 1A) and body weights (Fig.1B) in the various non-Ox groups during the experimental period. Non-fasting blood glucose concentrations in the Px OLETF rats began to increase on the second day, and remained at concentrations of about $10 \mathrm{mmol} / \mathrm{l}$ 


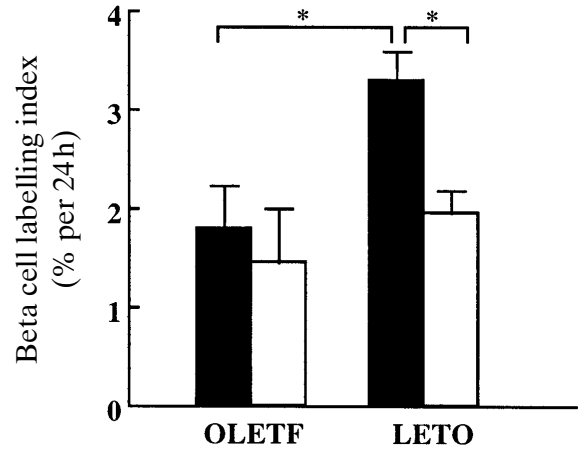

Fig.3. Proliferation of beta-cells in the Px rats $(\square)$ and sham ( $\square$ ) rats at 7 days after surgery, as quantified by immunostaining the cells for incorporation of BrdU into newly synthesized DNA of beta cells. The values are means \pm SEM. BrdU was injected intraperitoneally $(100 \mathrm{mg} / \mathrm{kg}) 4 \mathrm{~h}$ before the rats were killed. The animals used for data were those used for data in Table $1 . * p<0.01$

within the first week after surgery. Subsequently, the blood glucose values gradually decreased and remained at the concentrations present in the sham rats. The body weight increase in the Px OLETF female rats was significantly slower in the first 2 weeks after surgery. In contrast to the OLETF rats, no hyperglycaemia and slow body weight gain were observed in the Px LETO female rats after surgery. Figure 2 shows the effects of early Ox and $70 \%$ Px on blood glucose concentrations (Fig.2A) and body weights (Fig. 2B). In the Ox OLETF rats, a significant hyperglycaemia in each Px group was detected within the first week after surgery. It should be noted that the sustained hyperglycaemia in the testosterone replacement Px rats was dramatically higher than that in the other Px rats. A week later, the blood glucose concentrations in the Px rats decreased and, remained at the concentration of the sham counterparts for the remainder of the experiment, except for the testosterone replacement Px rats, in which the blood glucose values continued to be maintained at significantly higher concentrations than those of all other groups. Body weight (Fig. 2B) was significantly influenced by hormone replacement, showing that body weight gain was significantly slower in the estradiol replacement Px rats in the last 2 weeks of the experimental period, but were statistically faster for the testosterone replacement Px rats during the period of hormone administration.

Proliferation of beta-cells. BrdU-labelled beta cells in the remnant and remnant equivalent pancreas from the non-Ox rats are shown in Figure 3. The mean labelling index (LI) for beta cells in the remnant of the Px LETO rats was 1.7-fold higher for the sham counterparts, but this significantly increased LI was not evident in the remnant from the Px OLETF at 7 days after surgery. The mean beta-cell LI in the Px OLETF rats was significantly lower than that in the
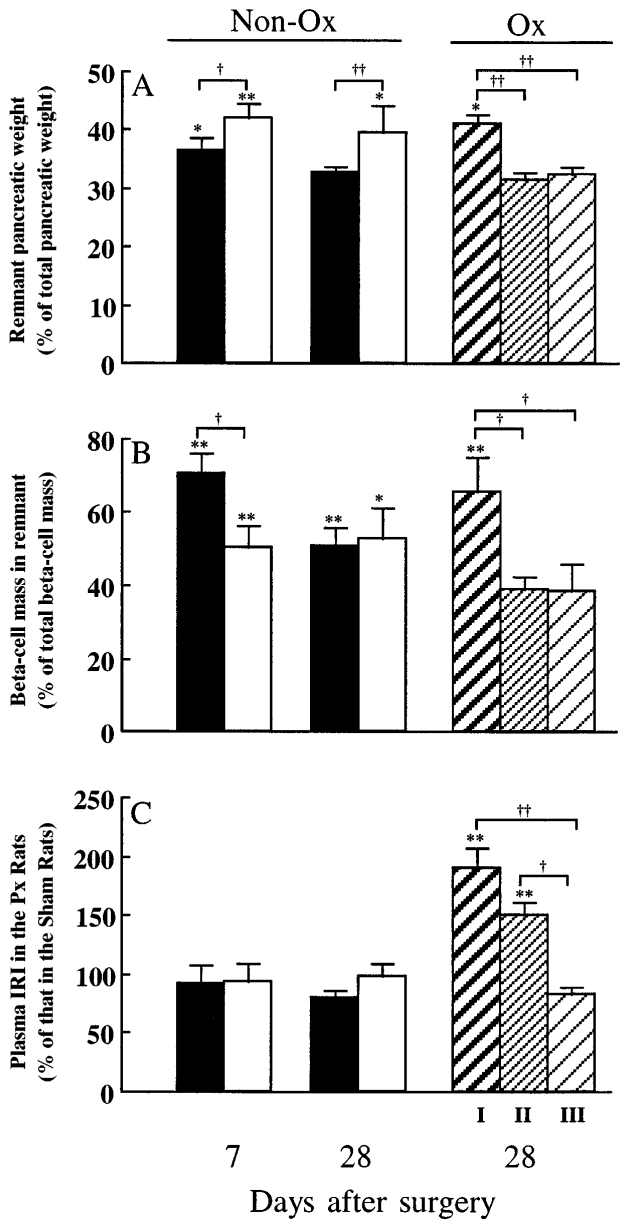

Fig. 4. Tissue weight (A), beta-cell mass (B) in remnant pancreas and plasma IRI levels $(\mathrm{C})$ in the non-Ox Px OLETF (口) and counterpart control LETO $(\square)$, and in addition, in the Ox-Px OLETF rats with estradiol valerate (I), testosterone enanthate (II) and vehicle (III) replacement after surgery. Values are expressed as the mean percentage of those in remnant pancreas from the Px rats to the whole pancreas from the counterpart sham rats. The bars represent SEM. The protocol used and doses of estradiol and testosterone, as well as the animals used for data are the same for Table $1 . * p<0.05, * * p<0.01$ vs the remnant equivalent from their counterpart sham rats; $\dagger p<0.05, \dagger \dagger p<0.01$

Px LETO rats $(1.80 \pm 42 \%$ per $24 \mathrm{~h}$ in the Px OLETF rats vs $3.39 \pm 52 \%$ per $24 \mathrm{~h}$ in the Px LETO, $p<0.01)$.

Tissue weight and beta-cell mass in remnant pancreas and fasting plasma IRI level. Tissue weight and betacell mass in the remnant pancreas, as well as plasma IRI concentration, as percentages of total values for whole pancreas, are shown in Figure 4. Taking into account the influence of body weight, the remnant pancreatic weight, as a percentage of the whole pancreas was adjusted for a ratio of body weight from counterpart sham rats, if significant difference in body weight at the end of the experiment was evident between Px rats and their shams. The remnant equi- 
Table 1. Characteristics of beta cells in the non-ovariectomized OLETF (F) and LETO (O), and the ovariectomized OLETF rats after a $70 \%$ pancreatectomy

\begin{tabular}{lll}
\hline Animals n & $\begin{array}{l}\text { Individual } \\
\text { beta-cell area } \\
\left(\mu \mathrm{m}^{2}\right)\end{array}$ & $\begin{array}{l}\text { Beta-cell mass/ } \\
\text { islet mass } \\
(\%)\end{array}$ \\
\hline 7 days after surgery & $\begin{array}{l}266.6 \pm 11.3^{\mathrm{b}} \\
\text { F-Px 6 }\end{array}$ & $82.1 \pm 0.7$ \\
F-sham 6 & $190.3 \pm 3.9$ & $85.5 \pm 3.4$ \\
O-Px 4 & $214.4 \pm 9.9$ & $79.9 \pm 2.9$ \\
O-sham 4 & $188.3 \pm 7.3$ & $82.5 \pm 3.7$ \\
28 days after surgery & & \\
F-Px 9 & $230.3 \pm 11.6^{\mathrm{b}}$ & $85.9 \pm 1.3$ \\
F-sham 6 & $181.0 \pm 9.2$ & $87.5 \pm 1.6$ \\
O-Px 4 & $202.7 \pm 9.7$ & $80.4 \pm 4.1$ \\
O-sham 4 & $174.6 \pm 10.5$ & $80.2 \pm 5.1$ \\
F-Ox + Px 8 & $216.9 \pm 7.6^{\text {ac }}$ & $79.5 \pm 1.3$ \\
F-Ox + Px + estradiol 8 & $250.6 \pm 9.4^{\mathrm{b}}$ & $85.8 \pm 1.5$ \\
F-Ox + Px + testosterone 7 & $219.8 \pm 11.3^{\text {ad }}$ & $78.8 \pm 2.3^{\mathrm{c}}$ \\
F-Ox + sham 8 & $191.7 \pm 7.0$ & $81.1 \pm 2.8$ \\
\hline Data are means SEM. Estradi
\end{tabular}

Data are means \pm SEM. Estradiol valerate $(50 \mu \mathrm{g} / \mathrm{kg} \mathrm{BW})$ was given at $48 \mathrm{~h}$ intervals and testosterone enanthate $(1 \mathrm{mg} / \mathrm{kg}$ BW) was given at weekly intervals. Injections were initiated on day 3 after surgery and continued until 1 week prior to killing. ${ }^{\mathrm{a}} p<0.05,{ }^{\mathrm{b}} p<0.01$ vs the remnant equivalent from the sham counterparts; ${ }^{\mathrm{c}} p<0.05,{ }^{\mathrm{d}} p<0.01$ vs Px + estradiol

valent weight in each sham group was approximately $30 \%$ of the values for the whole pancreas, suggesting that the relative growth of the remnant equivalent was similar to that of the remainder of the pancreas from the shams. The remnant weight (Fig. 4A) from the non-Ox Px rats of both OLETF and LETO, as a percentage of the whole pancreatic weight, showed an apparent increased growth $(36.5 \pm 2.1 \%$ and $32.6 \pm 0.9 \%$ in the Px OLETF vs $42.1 \pm 2.3 \%$ and $39.6 \pm 4.7 \%$ in the Px LETO at 7 days and 28 days after Px). However, such an increased growth of remnant pancreas in the Ox-Px OLETF rats was not evident, and remained at approximately $30 \%$ of total pancreatic weight, except for the estradiol replacement Px rats, in which the remnant pancreas increased to $41 \%$ of their sham counterparts. When the beta-cell mass $(\mathrm{mg})$, as a product of the relative volume of beta-cell mass (\%) and tissue weight, was calculated, a similar trend toward compensatory growth of beta-cell mass, as shown in Figure 4B, was found during the period of observation in the nonOx Px rats of both OLETF and LETO and also in the estradiol replacement Ox-Px OLETF rats. The fasting plasma IRI (Fig. 4C), not only in the non-Ox Px rats, but also in the Ox-Px rats, in the fasting state, approached the values for their sham counterparts, over $80 \%$ of the total IRI. It is noteworthy that hormone replacement caused a significant increase in plasma IRI levels $(190 \pm 16 \%$ of total plasma IRI value for the shams in the estradiol replacement Px rats and $151 \pm 9 \%$ of total IRI total plasma IRI value in the testosterone replacement Px rats).
Morphometric configurations of the beta cells. The mean area of individual beta cells was significantly enlarged in the non-Ox Px OLETF rats at both days 7 and 28 after surgery, as compared with their sham counterparts (Table 1). The same tendency appeared in the Px LETO, but failed to reach a significant level. No significant difference in the ratio of beta-cell mass to islet mass (as a consequence of beta-cell degranulation, fibrosis and/or fewer beta cells) was noted between the non-Ox Px and sham counterparts of both OLETF and LETO rats. It was clear, however, that the estradiol replacement resulted in a significantly enlarged individual beta-cell area, as compared with their Ox-sham and other Ox-Px rats. Testosterone replacement led to a significantly decreased ratio of beta-cell mass to islet mass, compared with the estradiol replacement Px animals, whereas no significant change was observed in individual beta-cell area.

\section{Discussion}

These results suggest that the capacity for beta-cell proliferation after reduction in beta-cell mass was affected by both sex hormonal and genetic components, as evidenced by the finding that a $70 \% \mathrm{Px}$ led to a sustained hyperglycaemia within the first week after surgery in the non-Ox female OLETF rats, but that the hyperglycaemia was gradually ameliorated with increased growth of the beta-cell mass thereafter. However, no sustained hyperglycaemia occurred in the control LETO rats after Px. It has been suggested that beta cells, even in adults, have a far greater ability to respond to glucose stimulation with compensatory growth by enhanced replication and hypertrophy of individual cell than previously realized [11-14]. In our previous report, a 70\% Px results in persistent hyperglycaemia, which is associated with insufficient proliferation of beta cells in male OLETF rats, while no sustained hyperglycaemia occurred in the control Px LETO rats, because of a higher rate of beta-cell proliferation with a parallel increased insulin content [8]. This suggests that the pancreata from male OLETF rats become insufficient to compensate for an insulin resistance-induced [15] and hypertriglyceridaemia-mediated [16] increased demand for insulin. From the present study, ovarian hormones appear to play an important role in the homeostatic control of beta-cell mass, indicating that beta-cell mass in the remaining tissue increases to over $30 \%$ of the value of the sham rats in the non-Ox Px OLETF rats within 28 days. In contrast, the results, in relation to $\mathrm{Ox}$ and $\mathrm{Px}$, indicate that this compensatory increase in beta-cell mass was decreased by the Ox, but that the defect was, in part, reversed by ovarian hormone replacement. It has been suggested that reduction in beta-cell mass is a critical pathological feature of pancreatic islets in Type II diabetes, that 
this is associated with impaired glucose tolerance and that problems with potentiation are secondary to inadequate beta-cell mass [7]. Our study clearly shows that the amount of beta-cell mass in the pancreata from both non-Ox and Ox with estradiol replacement Px OLETF rats were regulated by ovarian hormones (a 1.7-fold increase at 28 days after Px in the former and a 2.3-fold increase in the latter, compared with their sham counterparts), and this increased betacell mass functioned similarly to the resected betacell mass found in partially pancreatectomized rats [14]. This may provide a reasonable explanation for why enhanced beta-cell mass is a factor related to the preventative effect of ovarian hormones in the development of diabetes mellitus in this model rat.

It is noteworthy that ovarian hormones resulted in a significant hypertrophy of individual beta cells after surgery. The data indicate that increased betacell mass might be attributed to increased individual beta-cell area (beta-cell hypertrophy) rather than the expansion of beta-cell population, since the mean LI for beta cells in the Px OLETF rats was significantly lower than that in the control LETO rats. The results of several studies have suggested that genetic background is of considerable importance in determining the proliferative response of beta cells to diabetogenic factors $[17,18]$. Moreover, studies in normal and experimentally diabetic rats have shown that ovarian hormones possess the ability to increase beta-cell proliferation [19]. In fact, beta-cell hypertrophy accounted for much of the increased betacell mass, but an increase in the mitotic activity of beta cells early after surgery is not trivial and certainly is a significant component, since it would determine the function of latter remnant pancreas $[9,20]$. We also found that the Px-OLETF female rats had a higher BrdU-LI for beta cells $(1.8 \% / 24 \mathrm{~h})$ on day 7 after surgery than the male counterparts $(0.4 \% /$ $24 \mathrm{~h}$ ) [8], suggesting that basal beta-cell proliferation would be higher in females in this rat strain. However, the fact that the poor capacity for the expansion of beta-cell population to respond to the changes in functional demand caused by a $70 \%$ Px is clearly a genetic predisposition in this model rat. Since the results regarding BrdU-LI for beta cells in our previous report [8] suggests that the crucial period for expansion of the beta-cell population in this model rat is limited to the first 7 days after surgery, we only chose the 7th day after Px to test BrdU-LI for beta cells in this study. In integrating the findings of the present study into one hypothesis, we conclude that ovarian hormone-induced beta-cell hypertrophy may typically occur rather than expansion of beta-cell population to compensate for the burden on beta cells of the remnant pancreas in the Px female OLETF rats, whereas an increase in both BrdU-LI for beta cells and beta-cell hypertrophy early after surgery may be a critical component in the Px LETO rats.
Recently, several lines of evidence have been reported which show that pancreatic transcription factor, PDX-1/IPF-1/STF-1/IDX-1, plays an essential role in the progression of beta-cell proliferation [21, 22]. The evidence for an increased IDX-1(PDX-1/ IPF-1/STF-1) expression in the remnant pancreas during the first week after Px [23], and insulin-positive duct epithelial cells and some non-hormone cells which are strongly positive for PDX-1 in beta-cell regeneration model [24], suggest that it is likely that the expansion of beta-cell population, which occurs soon after Px can be attributed to the amount of some transcription factor and its expression pattern. Our unpublished data showed that PDX-1 expression in the islets from 8 week old male OLETF rats was significantly lower than that from the LETO counterparts. However, the issue of whether PDX-1/IPF-1/STF-1/ IDX-1 expression is regulated by ovarian hormones is not known. Regardless of the mechanisms of betacell proliferation responsible for ovarian hormones, reduced beta-cell apoptosis or cell loss may contribute to beta-cell mass homeostasis, a complex balance process of cell renewal and cell death. It has been reported that ovarian hormones prevent apoptosis in a rat endometrial cell line [25], but our knowledge of whether similar mechanisms by which ovarian hormones control cell death in pancreatic cells is minimal.

It has been reported that both $\mathrm{Ox}$ and testosterone replacement produce excessive body weight and insulin resistance in female OLETF rats [3]. It thus seems reasonable to suggest that the increase in plasma insulin concentration may represent a compensatory effort on the remnant pancreas for overcoming the loss of normal insulin sensitivity, and that the increasing demand for insulin may require the beta cells to secrete more insulin. This increased demand, in combination with a defect in beta-cell proliferation in the model rats used in this study, eventually may lead to beta-cell "exhaustion", evidenced by reduced beta-cell mass with a decreased ratio of beta-cell mass to islet mass (as a result of beta-cell degranulation and fibrous islets), but this process is inhibited by ovarian hormones, since increased plasma IRI levels were accompanied by a parallel increase in beta-cell mass in various nonOx Px and Ox with estradiol replacement Px rats.

In conclusion, ovarian hormones led to a beneficial effect on pancreatic beta cells per se in OLETF female rats. This was reflected by an increased growth in the pancreas accompanied by an increased beta-cell mass. The increased beta-cell mass, however, contributed to ovarian hormone-induced betacell hypertrophy rather than an increase in beta-cell population. The findings of the present study suggest that ovarian hormone-induced beta-cell hypertrophy, rather than beta-cell hyperplasia, may typically occur to compensate for the changes in functional demand caused by a $70 \%$ pancreatectomy in female OLETF rats. 
Acknowledgements. This work was supported, in part, by a Grant-in Aid for Scientific Research (No. 07671142) from the Ministry of Education, Science and Culture and by a grant for a 5-year project for the Exploration of the Pathogenesis of Diabetes Mellitus sponsored by Otsuka Pharmaceutical Co.

\section{References}

1. Kawano K, Hirashima T, Mori S, Saitoh Y, Kurosumi M, Natori T (1992) Spontaneous long-term hyperglycaemic rat with diabetic complications: Otsuka Long-Evans Tokushima Fatty (OLETF) strain. Diabetes 41: 1422-1428

2. Hirashima K, Kawano S, Mori K, Matsumoto TN (1995) A diabetogenic gene (ODB-1) assigned to the $\mathrm{x}$-chromosome in OLETF rats. Diabetes Res Clin Pract 27: 91-96

3. Shi K, Mizuno A, Sano T, Ishida K, Shima K (1994) Sexual difference in the incidence of diabetes mellitus in OtsukaLong-Evans-Tokushima-Fatty rats: effects of castration and sex hormone replacement on its incidence. Metabolism 43: $1214-1220$

4. Bailey CJ, Ahmed-Sorour H (1980) Role of ovarian hormones in the long-term control of glucose homeostasis. Diabetologia 19: 475-481

5. Bailey CJ, Matty AJ (1972) Glucose tolerance and plasma insulin of the rat in relation to the oestrous cycle and sex hormones. Horm Metab Res 4: 266-270

6. Yki-Jarvinen H (1984) Sex and insulin sensitivity. Metabolism 33: 1011-1015

7. Weir GC, Bonner-Weir S, Leahy JL (1990) Islet mass and function in diabetes and transplantation. Diabetes 39: 401-405

8. Zhu M, Noma Y, Mizuno A, Sano T, Shima K (1996) Poor capacity for proliferation of pancreatic $\beta$-cells in Otsuka Long-Evans Tokushima Fatty rat. A model of spontaneous NIDDM. Diabetes 45: 961-964

9. Bonner-Weir S, Baxter LA, Schuppin GT, Smith FE (1993) A second pathway for regeneration of adult exocrine and endocrine pancreas. A possible recapitulation of embryonic development. Diabetes 42: 1715-1720

10. Weibel ER (1963) Principles and methods for the morphometric studies of the lung and other organs. Lab Invest 12: 131-155

11. Swenne I (1982) The role of glucose in vitro regulation of cell cycle kinetics and proliferation of fetal pancreatic $\beta$ cells. Diabetes 31: 754-760

12. McDowell EM, DeSanti AM, Strum JM (1989) Correlations between blood glucose levels and Bromodeoxyuri- dine labeling indices of pancreatic islet cells forming streptozotocin administration to pregnant Syrian golden hamsters. Virchows Archiv B Cell Pathol 57: 223-230

13. Bonner-Weir S, Deery D, Leahy JL, Weir GC (1989) Compensatory growth of pancreatic $\beta$-cells in adult rats after short-term glucose infusion. Diabetes 38: 49-53

14. Bonner Weir S, Trent DF, Weir GC (1983) Partial pancreatectomy in rat and subsequent defect in glucose-induced insulin release. J Clin Invest 71: 1544-1553

15. Ishida K, Mizuno A, Zhu M, Sano T, Shima K (1995) Which is the primary etiological event in Otsuka LongEvans Tokushima Fatty rat, a model of spontaneous noninsulin dependent diabetes mellitus, insulin resistance, or impaired insulin secretion? Metabolism 44: 940-945

16. Man Z-W, Zhu M, Noma Y et al. (1997) Impaired $\beta$-cell function and desposition of fat droplets in pancreas as a consequence of hypertriglyceridaemia in OLETF rat, a model of spontaneous non-insulin-dependent diabetes mellitus (NIDDM). Diabetes 46: 1718-1724

17. Swenne I, Andersson A (1984) Effect of genetic background on the capacity for islet cell replication in mice. Diabetologia 27: 464-467

18. Andersson A (1983) The influence of hyperglycaemia, hyperinsulinaemia and genetic background on the fate of intrasplenically implanted mouse islets. Diabetologia 25: 269-272

19. Houssy BA (1951) Action of sex hormones on experimental diabetes. BMJ II:505-510

20. Finegood DT, Scaglia L, Bonner-Weir S (1995) Dynamics of $\beta$-cell mass in the growing rat pancreas. Estimation with a simple mathematical model. Diabetes 44: 249-256

21. Jonsson J, Carlsson L, Edlund T, Edlund H (1994) Insulinpromoter-factor 1 is required for pancreas development in mice. Nature 371: 606-609

22. Offield MF, Jetton TL, Labosky PA et al. (1996) PDX-1 is required for pancreatic outgrowth and differentiation of the rostrol duodenum. Development 122: 983-995

23. Zangen DH, Bonner-Weir S, Lee CH et al. (1997) Reduced insulin, GLUT2, and IDX-1 in $\beta$-cells after partial pancreatectomy. Diabetes 46: 258-264

24. Waguri M, Yamamoto K, Miyagawa JI et al. (1997) Demonstration of two different processes of $\beta$-cell regeneration in a new diabetic mouse model induced by selective perfusion of alloxan. Diabetes 46: 1281-1290

25. Pecci A, Scholz A, Pelster D, Beato M (1997) Progestins prevent apoptosis in a rat endometrial cell line and increase the ratio of bcl-X-L to bcl-X-S. J Biol Chem 272: 11791-11798 